\title{
Diabetic ketoacidosis complicated by emphysematous pyelonephritis: a case report and literature review
}

\author{
Yuanhao Song and Xingping Shen * (D)
}

\begin{abstract}
Background: The management of emphysematous pyelonephritis (EPN) includes conservative medical treatment, percutaneous drainage, and surgical resection of the involved kidney. EPN with diabetic ketoacidosis(DKA) is very rare, in which the clinical management of refusing surgical drainage is inexperienced.

Case presentation: A 34-year-old woman presented with abdominal pain, chills, fever, nausea, vomiting, chest tightness, and shortness of breath. Blood test results were consistent with diabetic ketoacidosis. Urinary computed tomography scan showed multiple stones in the right kidney and lower ureter, with right hydronephrosis. Blood culture demonstrated Escherichia coli bacteremia, and EPN was diagnosed. Considering the need for a second percutaneous nephrolithotomy, the patient refused percutaneous drainage. After continuous intravenous infusion of small doses of insulin and antibiotic treatment, the ketoacidosis resolved. The patient's temperature returned to normal and abdominal pain was alleviated, and liver and kidney functions were also back to normal. After hospital discharge, the patient underwent two percutaneous nephrolithotomy in the department of urology.

Conclusions: EPN with diabetic ketoacidosis should be diagnosed as soon as possible. For patients with Class 1 and Class 2 EPN with diabetic ketoacidosis and urinary tract obstruction, if surgical drainage is refused, it is particularly important to rapidly correct diabetic ketoacidosis and intravenous use of sensitive antibiotics, so as to create conditions for follow-up percutaneous nephrolithotomy.
\end{abstract}

Keywords: Emphysematous pyelonephritis, Diabetes, Diabetic ketoacidosis, Kidney stones, Antibiotics

\section{Background}

Emphysematous pyelonephritis (EPN) is an acute, severe necrotizing infection affecting renal parenchyma, collecting system as well as surrounding tissue with hallmark of presence of gas within these structures [1]. Several large-scale retrospective studies have shown that affected patients commonly presented with fever, abdominal pain, nausea, and vomiting [1,2]; although, occasionally, patients have experienced no obvious symptoms [3]. There has been no general consensus with respect to the diagnosis and treatment of EPN. A computed tomography (CT) scan is currently the gold-standard diagnostic test [4]. Treatment includes conservative medical therapy, with or without surgical drainage or nephrectomy [5].

\footnotetext{
* Correspondence: sxpsxzlg@qq.com

Department of Endocrinology, Zhongshan Hospital Xiamen University,

Xiamen 361004, Fujian, China
}

At present, EPN with diabetic ketoacidosis is rare, only a few cases have been reported [6-10]. And diabetic ketoacidosis is an important predictor of death in patients with EPN [11]. Effective surgical drainage is a key measure for the management of EPN with diabetic ketoacidosis and urinary tract obstruction. In this case, the clinical management of patients refusing surgical drainage is inexperienced.

\section{Case presentation}

A 34-year-old woman was admitted to the hospital due to abdominal pain, chills, fever, chest tightness, and shortness of breath after eating contaminated food 2 days earlier. After admission, she became nauseated and vomited; but without dysuria, urinary frequency, or urgency. She had a medical history of type I diabetes for 19 months, with poor control of blood glucose due to non-compliance with the insulin treatments. One month 
prior, she was found to have kidney stones. Physical examination revealed a temperature of $36.0^{\circ} \mathrm{C}$, pulse rate of 87 beats/minute, respiratory rate of 35 breaths/min, and a blood pressure of 105/66 mm of $\mathrm{Hg}$. She had shortness of breath, dry skin, and dry mucous membranes and tongue; although her lungs sounded clear. There was an abdominal wall muscle strain in the epigastric area and right upper quadrant, with obvious tenderness. The right costovertebral angle also had tenderness.

Laboratory tests and imaging studies were as follows. Blood tests after admission showed a white blood cell count of $21.08 \times 10^{9} / \mathrm{L}$, neutrophil count of $18.53 \times 10^{9} / \mathrm{L}$, neutrophil percentage of $87.9 \%$, platelet count of $107 \times$ $10^{9} / \mathrm{L}$; and concentrations for albumin of $37.42 \mathrm{~g} / \mathrm{L}$, glucose of $35.95 \mathrm{mmol} / \mathrm{L}$, urea of $10.54 \mathrm{mmol} / \mathrm{L}$, creatinine of $145.1 \mu \mathrm{mol} / \mathrm{L}$, bicarbonate of $2.2 \mathrm{mmol} / \mathrm{L}$, potassium of $6.60 \mathrm{mmol} / \mathrm{L}$, sodium of $122.10 \mathrm{mmol} / \mathrm{L}$, $\beta$-hydroxybutyric acid of $10.08 \mathrm{mmol} / \mathrm{L}$, lactic acid of $2.43 \mathrm{mmol} / \mathrm{L}, C$ reactive protein of $448.39 \mathrm{mg} / \mathrm{L}$, and procalcitonin > $100.000 \mathrm{ng} / \mathrm{mL}$. Blood gas analysis showed a $\mathrm{pH}$ of 6.984 , carbon dioxide partial pressure of $10.9 \mathrm{~mm}$ of $\mathrm{Hg}$, oxygen partial pressure of $138.2 \mathrm{~mm}$ of $\mathrm{Hg}$, measured bicarbonate of $2.5 \mathrm{mmol} / \mathrm{L}$, calculated bicarbonate of $6.4 \mathrm{mmol} / \mathrm{L}$, anion gap of $32.2 \mathrm{mmol} / \mathrm{L}$, base excess of $-27.2 \mathrm{mmol} / \mathrm{L}$, and carbon dioxide of $2.9 \mathrm{mmol} / \mathrm{L}$. Urinalysis measurements were glucose, 4+; ketone body, 4+; leukocyte esterase, weakly positive; and white blood cell number, $41 / \mu \mathrm{L}$; with negative results for urine culture. Blood culture identified Escherichia coli. Urinary CT scan revealed multiple stones in the right kidney and lower ureter, with right hydronephrosis; we therefore diagnosed EPN (Figs. 1, 2, and 3 reflect the CT scans during hospital admission).

After being admitted to the hospital for definite diagnosis, our endocrinology department cooperated with

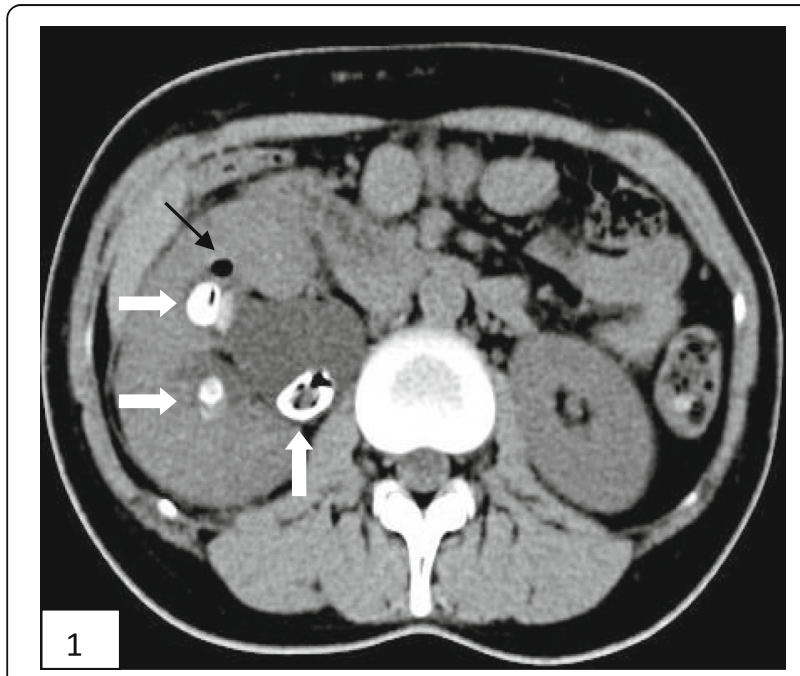

Fig. 1 Show the non-contrast urinary CT scans during hospital admission

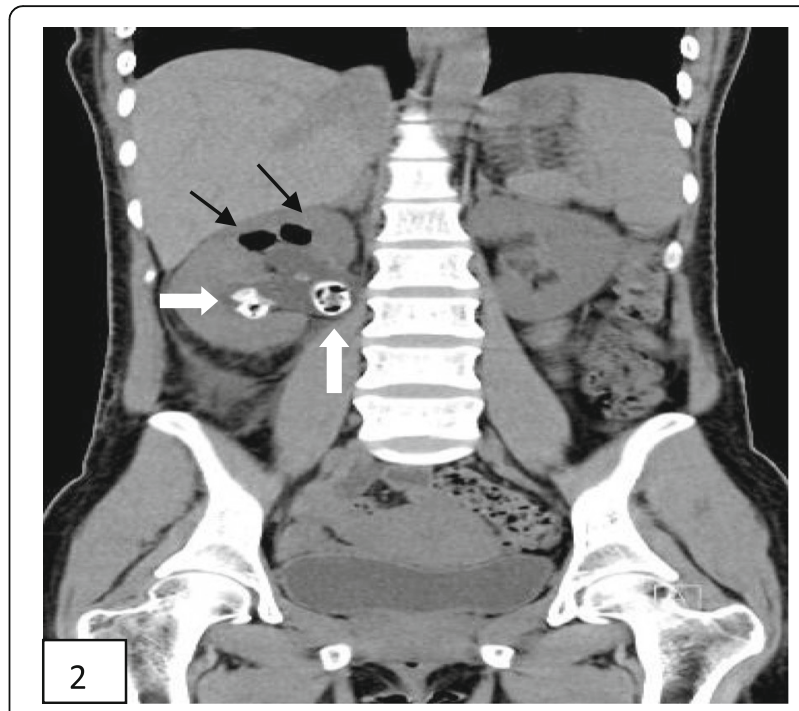

Fig. 2 Show the non-contrast urinary CT scans during hospital admission

urology, nephrology, infection department and imaging department to formulate a treatment plan, which suggested that the patients should undergo percutaneous drainage but the patient refused. Hospital management and outcomes after admission included intravenous infusion of small doses of insulin to correct ketoacidosis. Liver and renal functions returned to normal after albumin infusion and hydration. Based upon blood culture results, Cefoperazone sulbactam was upgraded to meropenem. Inflammatory indicators and body temperature gradually returned to normal, and abdominal pain was relieved. Repeated blood cultures were negative, and urine culture remained negative. Repeated urinary CT

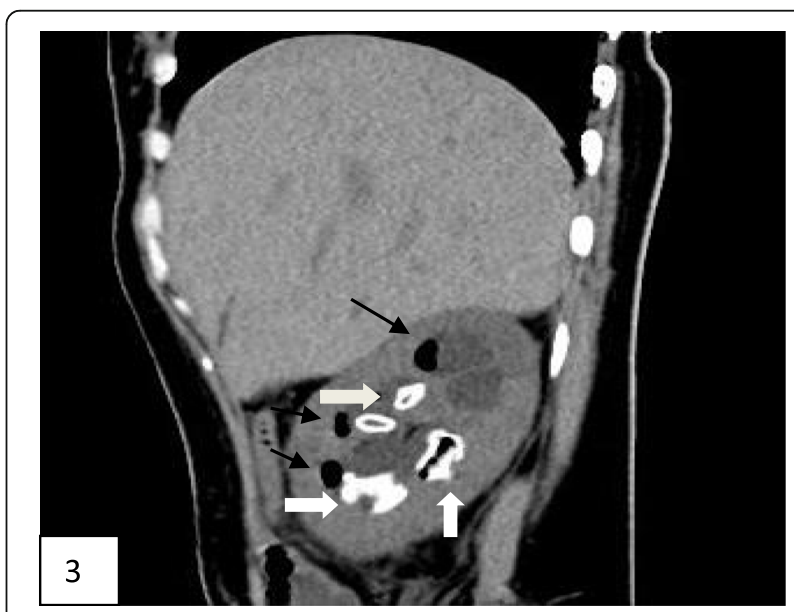

Fig. 3 Show the non-contrast urinary CT scans during hospital admission 
scans revealed multiple stones in the right kidney with worsening right hydronephrosis and pyelonephritis, but with reduced gas accumulation (Fig. 4).

One month after discharge, the patient underwent percutaneous nephrolithotomy in the department of urology, and the urinary CT was reexamined after operation (Fig. 5).Two months after discharge, the patient underwent the second percutaneous nephrolithotomy in the department of urology, and the urinary CT was reexamined after operation (Fig. 6). Currently, three months after discharge, clinical follow-up checks detect normal renal function and stable blood glucose control.

\section{Discussion and conclusions}

In 1898, Kelly and MacCullum reported the first case of kidney infection with gas accumulation [12]. In 1962, Schultz and Klorfein described this disorder as emphysematous pyelonephritis [13]. Since then, there have been reports of various types of kidney infection with gas accumulation.

The pathogenesis of emphysematous pyelonephritis (EPN) is still unclear. It is believed that multiple factors, including diabetes, elevated glucose levels in kidney tissue, urinary tract obstruction, impaired renal circulation, decreased host immune function, and the presence of gasproducing microbial infections could cause EPN [1, 14]. EPN most commonly affects diabetic patients [2, 15-17]; and it is currently believed that the increased susceptibility to developing EPN in diabetic patients is due to impaired renal tissue perfusion [18]. Additionally, high blood glucose concentrations can also promote anaerobic growth and gas-producing metabolism [18].

CT scan is now considered to be the best method to diagnose EPN, and the most commonly accepted CT classification system for EPN was proposed by Huang

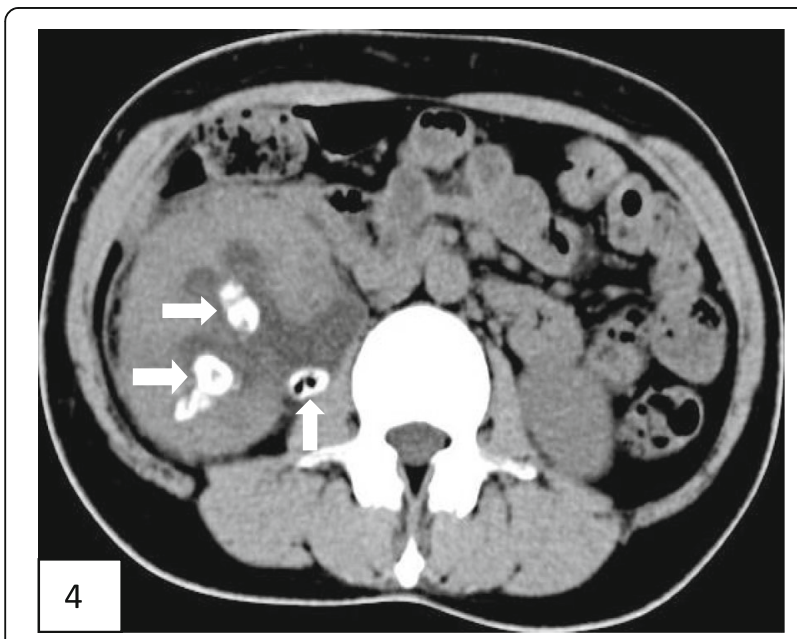

Fig. 4 Shows the repeat non-contrast $C T$ scan before hospital discharge and associates in 2000 [19]. These authors suggested 4 classes of EPN: Class 1, where gas is confined only to the collection; Class 2, where gas is located within the renal parenchyma and does not spread to the extrarenal space; Class 3A, where gas or an abscess spreads to the perinephric space; Class $3 \mathrm{~B}$, where gas or the abscess spreads to the pararenal space; and Class 4, depicting a bilateral or solitary kidney with EPN [19].. However, in one recent study, investigators attempted to re-correlate CT classification with clinical treatment options, analyzing 34 cases from 2009 to 2018. Their results showed that most Class 1 and Class 2 patients achieved satisfactory outcomes purely by conservative treatment. Only a small number of patients with urinary tract obstruction required combined percutaneous drainage, 2 of the 6 Class $3 \mathrm{~A}$ patients required nephrectomy, and 2 Class 3B patients also required nephrectomy [16].

$\mathrm{Lu}$ et al. retrospectively analyzed 51 patients with EPN and demonstrated that the most common bacteria were Escherichia coli, followed by Streptococcus pneumoniae, Enterococcus, and Pseudomonas aeruginosa; with a small number of patients showing mixed bacterial infections. The antibiotic-resistance rate was greater than $15 \%$ for fluoroquinolone, ampicillin, 1st- and 2nd-generation cephalosporins, and gentamicin; whereas the resistance rates for the 3rd- and 4th-generation cephalosporins were only 10.9 and $6.5 \%$, respectively. These authors concluded that the 3rd-generation cephalosporins could be used routinely for most affected patients. However, carbapenem antibiotics such as meropenem should be selected if a patient carries high risk factors, including recent hospitalization, antibiotic use, or DIC [20]. At the present time, most studies depict Escherichia coli as the most common pathogenic bacterium [1, 17]; although there have been recent reports of EPN caused by Candida albicans [21]. Therefore, selection of antibiotics should be based upon the local epidemiology and antibiotic- resistance patterns. In addition, multiple blood and urine cultures should be performed. It is extremely important to then revise the antibiotics according to the disease severity and the antibiotic-susceptibility test results.

There is recent evidence that patients who received conservative management achieved satisfactory results [15]. However, a study of 17 patients with EPN also showed that patients who received conservative treatment tend to experience acute increases in the Sequential Organ Failure Assessment (SOFA) score after the beginning of treatment, even if transient, which presents a great risk of septic mortality [15]. Adequate surgical drainage combined with intravenous antibiotics have resulted in satisfactory results in patients with EPN and multiple organ failure who were not appropriate candidates for nephrectomy [17, 22, 23]. In another study researchers tried to evaluate the clinical prognosis and 


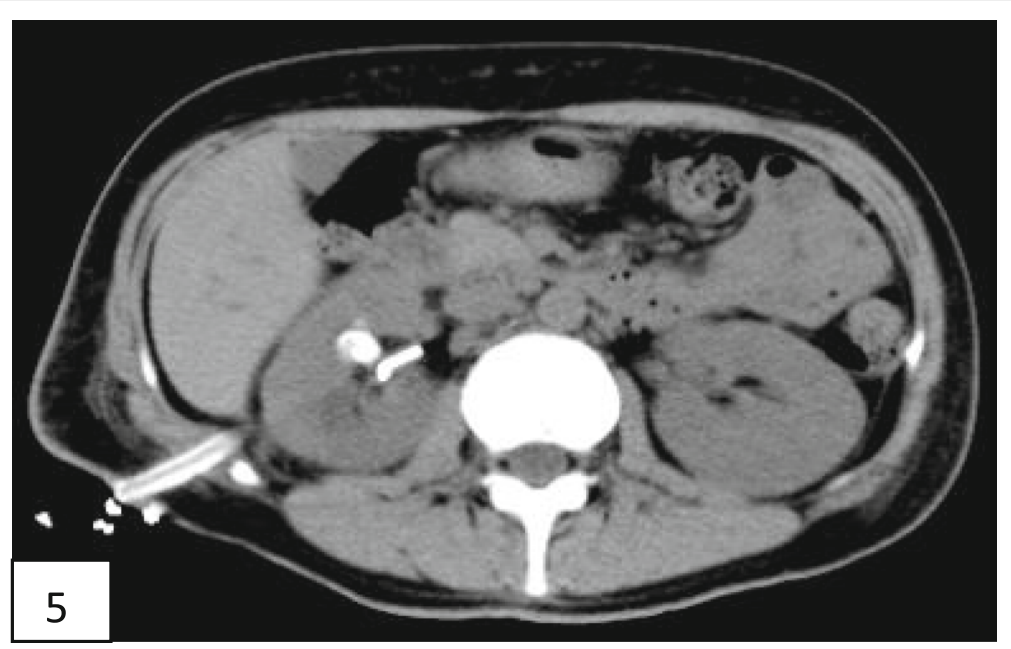

Fig. 5 Shows the repeat non-contrast CT scan after the first percutaneous nephroscopy

outcomes of this disorder by analyzing 74 patients with EPN [1]. Their results showed that 1 . fever was the most common clinical presentation, which was followed by lower back pain; 2. most patients had diabetes, which was followed by urolithiasis; 3. Escherichia coli was still the most common pathogen; 4. older age, high bodymass index, impaired renal function, thrombocytopenia, sensory changes, and shock were associated with a poor prognosis; and 5. treatment mainly consisted of rapid hydration, maintenance of electrolyte balance, use of systemic antibiotics, strict control of blood glucose, effective urine drainage, and nephrectomy if necessary [1]. Sanford et al. have shown that mild EPN was treatable by conservative management that included infectious agent-susceptible antibiotics [24]. However, patients with severe infections required percutaneous renal puncture drainage and/or double-J stenting [24].
Nanki et al. successfully treated a 58-year-old woman with EPN caused by Escherichia coli complicated with diabetic ketoacidosis through nephrectomy, antibiotics and recombinant human granulocyte colony-stimulating factor(rhG-CSF) [8]. Harrabi et al. reported a 64-yearold woman with EPN complicated with diabetic ketoacidosis who died of septic shock without surgical drainage [6]. However, another patient with EPN with diabetic ketoacidosis who underwent percutaneous renal drainage combined with antibiotics died 7 days after admission [9]; Unlike our patient, according to CT, this patient belongs to class 3A EPN. Recently, it has been reported that EPN with diabetic ketoacidosis in the patient with allogeneic renal transplantation improved after conservative treatment with intravenous antibiotics without percutaneous drainage or nephrectomy [10]; But our patient is different from this successful case in that there are urinary tract

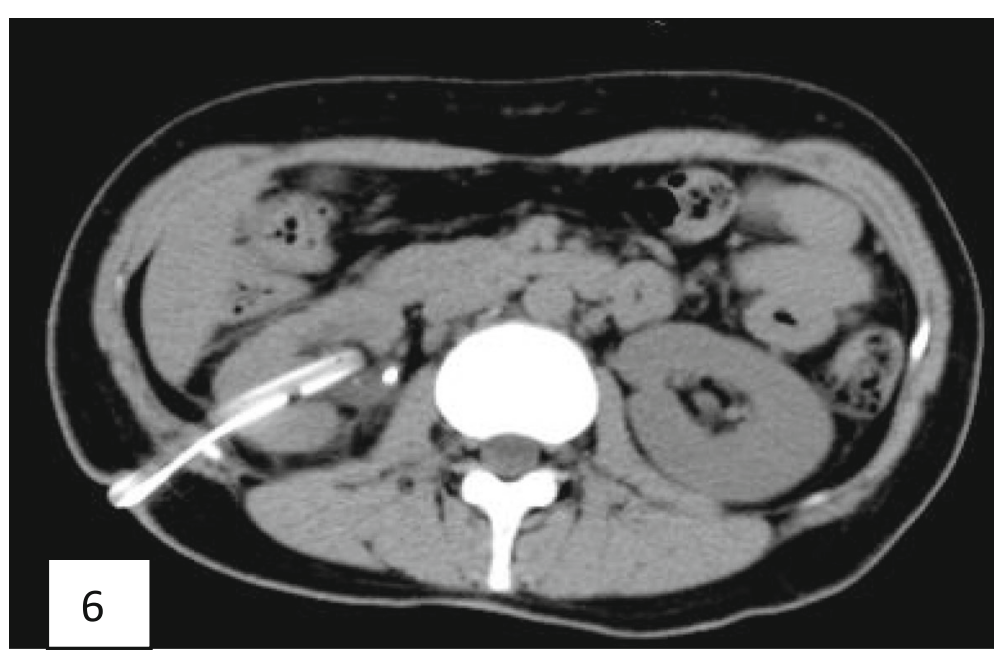

Fig. 6 Shows the repeat non-contrast CT scan after the second percutaneous nephroscopy 
obstruction factors such as right kidney stone and right ureteral stone, and percutaneous drainage is more important in the treatment.

Our successful management of this patient lies in the following points: 1 . We confirmed the diagnosis of EPN by urinary $\mathrm{CT}$ examination as soon as the patient was admitted to hospital; 2. Rapid correction of ketoacidosis by continuous intravenous infusion of low-dose insulin and fluid resuscitation; 3. We performed blood culture before using antibiotics and upgraded cefoperazone sulbactam to meropenem according to the results of blood culture and the antibiotic-susceptibility test results; 4 . Our endocrinology department collaborated with urology, nephrology, infection and imaging departments to develop a treatment plan; 5 . What is particularly important is that although the patient has sepsis, it belongs to Class $2 \mathrm{EPN}$. However, our treatment is also inadequate: the patient did not receive percutaneous drainage. Although the accumulation of gas in the kidney was less than before, the right hydronephrosis increased. In the end, the patient still underwent two percutaneous nephrolithotomy. Failed to achieve the goal that the patient wanted to have only one operation.

Our case report makes up for the gap in the clinical management of patients with EPN with diabetic ketoacidosis with urinary tract obstruction who refuse surgical drainage, but it is unclear whether our management experience can be extended to similar patients of EPN Class 3A,Class 3B and Class 4.

In conclusion, EPN with diabetic ketoacidosis should be diagnosed as soon as possible. For patients with Class 1 and Class 2 EPN with diabetic ketoacidosis and urinary tract obstruction, if surgical drainage is refused, it is particularly important to rapidly correct diabetic ketoacidosis and intravenous use of sensitive antibiotics, so as to create conditions for follow-up percutaneous nephrolithotomy.

\section{Abbreviations}

CT: Computed tomography; DKA: Diabetic ketoacidosis EPN: emphysematous pyelonephritis; RHG-CSF: Recombinant human granulocyte colony-stimulating factor; SOFA: Sequential Organ Failure Assessment

\section{Acknowledgements}

Not applicable.

\section{Authors' contributions}

YHS made contributions to data collection, literature search. XPS was responsible for the patient's diagnosis and treatment. YHS drafted original manuscript and XPS reviewed and edited the manuscript. All authors read and approved the final manuscript.

\section{Funding}

No funding was obtained for this study.

\section{Availability of data and materials}

The datasets used and analysed during the current study are available from the corresponding author on reasonable request.

All authors have read the paper and agree that it can be published.

\section{Ethics approval and consent to participate}

The study complied with the Declaration of Helsinki and was approved by the Institutional Ethics Committee of Zhongshan Hospital Xiamen University. (Xiamen, China). Written informed consents were obtained from all the study participants.

\section{Consent for publication}

Written informed consent was obtained from the patient for publication of this case report and any accompanying images.

\section{Competing interests}

The authors declare that they have no competing interests.

Received: 23 December 2019 Accepted: 15 January 2020

Published online: 29 January 2020

\section{References}

1. Sokhal AK, Kumar M, Purkait B, Jhanwar A, Singh K, Bansal A, et al. Emphysematous pyelonephritis: changing trend of clinical spectrum, pathogenesis, management and outcome. Turk J Urol. 2017:43(2):202-9.

2. Karthikeyan VS, Manohar CMS, Mallya A, Keshavamurthy R, Kamath AJ. Clinical profile and successful outcomes of conservative and minimally invasive treatment of emphysematous pyelonephritis. Cent European J Urol. 2018;71(2):228-33

3. Yeung $\mathrm{A}$, Cheng $\mathrm{CH}$, Chu $\mathrm{P}$, Man $\mathrm{CW}$, Chau $\mathrm{H}$. A rare case of asymptomatic emphysematous pyelonephritis. Urol Case Rep. 2019;26:100962.

4. Mahesh HR, Padala KP, Swamy K, Pailoor a. A rare case of Pneumoureter: emphysematous Pyelitis versus emphysematous pyelonephritis. J Clin Diagn Res. 2015:9(11):TD03-5.

5. Boakes E, Batura D. Deriving a management algorithm for emphysematous pyelonephritis: can we rely on minimally invasive strategies or should we be opting for earlier nephrectomy? Int Urol Nephrol. 2017:49(12):2127-36.

6. Harrabi H, Marrakchi C, Daoud E, Elleuch E, Hammami B, Maaloul I, et al. Bilateral emphysematous pyelonephritis caused by Candida glabrata: an exceptional entity. Nephrol Ther. 2010;6(6):541-3.

7. Kirpekar M, Cooke KS, Abiri MM, Lipset RE. US case of the day. Emphysematous pyelonephritis. Radiographics. 1997;17(6):1601-3.

8. Nanki T, Ageishi Y, Iwama T, Murase H, Uchikawa T. Emphysematous pyelonephritis successfully treated with nephrectomy and granulocyte colony-stimulating factor. Intern Med. 1994;33(4):234-6.

9. Ghosh GC, Sharma B, Kamble U, Lamba BM. Vanishing kidney disease. BMJ Case Rep. 2013. https://doi.org/10.1136/bcr-2013-009117.

10. Crouter AJ, Abraham MK, Wilkerson RG. Emphysematous pyelonephritis in a renal allograft. Am J Emerg Med. 2017;35(3):520 e1- e2.

11. Wang JM, Lim HK, Pang KK. Emphysematous pyelonephritis. Scand J Urol Nephrol. 2007;41(3):223-9.

12. Kelly HA, WG MC. Pneumaturia. JAMA. 1898;31:375-81.

13. Schultz EH Jr, Klorfein EH. Emphysematous pyelonephritis. J Urol. 1962;87:762-6.

14. Arsene C, Saste A, Arul S, Mestrovich J, Kammo R, Elbashir M, et al. A case series of emphysematous pyelonephritis. Case Rep Med. 2014;2014:587926.

15. Ozawa M, Ichiyanagi O, Fujita S, Naito S, Fukuhara H, Suenaga S, et al. Risk of SOFA deterioration in conservative treatment for emphysematous pyelonephritis: pitfalls of current trends in therapeutics from multicenter clinical experience. Curr Urol. 2019;12(3):134-41.

16. Elawdy MM, Osman Y, Abouelkheir RT, El-Halwagy S, Awad B, El-Mekresh M. Emphysematous pyelonephritis treatment strategies in correlation to the $C T$ classification: have the current experience and prognosis changed? Int Urol Nephrol. 2019:1709-13.

17. Kangjam SM, Irom KS, Khumallambam IS, Sinam RS. Role of conservative Management in Emphysematous Pyelonephritis - a retrospective study. J Clin Diagn Res. 2015;9(11):PC09-11.

18. Eswarappa M, Suryadevara S, John MM, Kumar M, Reddy SB, Suhail M. Emphysematous pyelonephritis case series from South India. Kidney Int Rep. 2018;3(4):950-5.

19. Huang JJ, Tseng CC. Emphysematous pyelonephritis: Clinicoradiological classification, management, prognosis, and pathogenesis. Arch Intern Med. 2000;160(6):797-805.

20. Lu YC, Hong JH, Chiang BJ, Pong YH, Hsueh PR, Huang CY, et al. Recommended initial antimicrobial therapy for emphysematous 
pyelonephritis: 51 cases and 14-year-experience of a tertiary referral center Medicine. 2016;95(21):e3573.

21. Ideguchi S, Yamamoto K, Ikeda A, Hashimoto K, Takazono T, Saijo T, et al. A case of bilateral emphysematous pyelonephritis caused by Candida albicans. J Infect Chemother. 2019;25(4):302-6.

22. Deoraj S, Zakharious F, Nasim A, Missouris C. Emphysematous pyelonephritis: outcomes of conservative management and literature review. BMJ Case Rep. 2018:bcr-2018-225931.

23. Tilden W, Valliani S, Chana HS. Severe emphysematous pyelonephritis: conservative management plus image-guided percutaneous drainage prior to successful elective nephrectomy. A case report and review of the literature. BJR Case Rep. 2015;1(1):20140004.

24. Sanford TH, Myers F, Chi T, Bagga HS, Taylor AG, Stoller ML.

Emphysematous pyelonephritis: the impact of urolithiasis on disease severity. Transl Androl Urol. 2016:5(5):774-9.

\section{Publisher's Note}

Springer Nature remains neutral with regard to jurisdictional claims in published maps and institutional affiliations.

Ready to submit your research? Choose BMC and benefit from:

- fast, convenient online submission

- thorough peer review by experienced researchers in your field

- rapid publication on acceptance

- support for research data, including large and complex data types

- gold Open Access which fosters wider collaboration and increased citations

- maximum visibility for your research: over $100 \mathrm{M}$ website views per year

At BMC, research is always in progress.

Learn more biomedcentral.com/submissions 